\title{
Design and Implementation on Credit Card Risk Control System
}

\author{
Jianmei Ma \\ College of Finance and Trade, Bohai University, Jinzhou, 121013, China \\ majianmei0521@126.com
}

Keywords: credit card; risk control system; design and implementation; Value at Risk

\begin{abstract}
With the great-leap-forward development of credit card, risk is also showing a trend of gradual expansion. Based on pre-control methods of damage control and Visual Basic .NET, the paper designs and implements credit card risk control system to provide information means for credit card risk control. Research bases on credit risk management model, first of all, analyzed and designed the system function structure consisting of "Main routine function, Analysis control function and Supporting data" and the like; and then, created business logic components, the data model and object model for "Credit card limit control" was designed; finally, studying the implementation of presentation layer, the main controls and property settings for "Credit limit control" form was designed. The system can ensure sustained and healthy development of credit card business and maintain stability of financial sector.
\end{abstract}

\section{Introduction}

Credit card is issued to customers with a stable income or good economic foundation by financial institutions in accordance with the line of credit, which uses non-cash payment consumption pattern with a simple credit services. Credit card is likely to bring income to financial institutions directly, such as annual fee, deposit spreads, specially engaged fees and overdraft interest, which is an important profit source of financial institutions. Credit card brings high returns for financial institutions, but also there is a huge risk that some customers overdraft maliciously and refuse to repay, caused huge losses to the financial institutions. How to prevent and control the risks are pressing problems for financial institutions [1-3].

Risk managers take various measures and methods to eliminate or reduce various possibilities of risk events, or to reduce the losses caused by the risk events, which is risk control. Risk control includes four basic ways, namely risk avoidance, loss control, risk transfer and risk retention. The loss control is not to give up risk, but to develop plans and take measures to reduce the possibility of loss or reduce the actual loss. Control stage includes pre-control, mid-control and after control, the three stages. The main purpose of pre-control is to reduce the probability of loss, mid-control and after control is to reduce the actual loss. Based on pre-control methods of damage control and Visual Basic .NET, the paper designs and implements credit card risk control system to provide information means for credit card risk control.

\section{Credit Risk Management Model}

VaR, known as value at risk model, commonly is used for risk management in financial institutions. VaR applies to measure various market risks comprehensively, including interest rate risk, exchange rate risk, equity risk, commodity price risk and derivative financial instruments risk. Financial institutions can use a specific value VaR to reflect the risk profile of the financial institution or investment portfolio, which can facilitate the exchange of the various business units on risk information, but also to facilitate organization's senior management to keep abreast of the overall risk profile, so it is conducive for financial institutions to manage risks unified. The general formula of $\operatorname{VaR}$ is:

$$
P(\Delta P \Delta t \leq V a R)=\alpha
$$

In the above formula, $P$ represents the probability that loss of asset value is less than the upper 
limit of the potential loss, which is Probability in English; $\Delta P$ represents the value losses of a certain financial asset in a holding period $\Delta t$; $V a R$ is value at risk under the given confidence level $\alpha$, that the possible loss-limit; $\alpha$ represent a given confidence level.

Credit risk management of credit card can use VaR model. Set time limit of risk is $t$, customers with credit cards are represented by $M, c_{i}$ represents $i$-th customer $\left(c_{i} \in M\right)$. For each customer $c_{i}$, the probability of default is $d_{i}$, loss given default is $t_{i}$; if the customer does not default, the bank's earning is $m_{i}$. Bank's loss function is defined as:

$$
f(x, y)=\sum_{i=1}^{n}\left[d_{i} t_{i}-\left(1-d_{i}\right) m_{i}\right]=(x-x-t) y
$$

Wherein, $\quad x=\left(d_{1}, d_{2}, \mathrm{~L}, d_{N}\right) \quad, \quad t=(1,1, \mathrm{~L}, 1) \quad$ are $\quad N$-dimensional vector, $y=\left(t_{1}, t_{2}, \mathrm{~L}, t_{N}, m_{1}, m_{2}, \mathrm{~L}, m_{N}\right)^{T}$.

Set $x$ is determined vector, subject to the multidimensional normal distribution $N_{2 N}(W, B), \mu$ is $2 N \times 1$ vector, $\sigma$ is $2 N \times 2 N$ vector, the loss function $f(x, y)$ subjects to normal distribution, you can obtain $V a R$ of the loss function.

$$
\begin{gathered}
\operatorname{Prob}[f(x, y)] \mathrm{f} \operatorname{VaR}=1-c \\
\operatorname{Pr} o b\left[\frac{f(x, y)-(x, x-t) W}{\sqrt{(x, x-t) B(x, x-t)^{T}}}\right] \mathrm{f} \frac{V a R-(x, x-t) W}{\sqrt{(x, x-t) B(x, x-t)^{T}}}=1-c \\
\operatorname{VaR}=Z_{c} \sqrt{(x, x-t) B(x, x-t)^{T}}+(x, x-t) W
\end{gathered}
$$

Wherein, $c$ represents confidence level, $Z_{c}$ represents the quantile of standard normal distribution $c$.

After calculating $V a R$ value, if the $V a R$ value is too large, we can examine strictly, control the number of cards, timely track and monitor in the consumption process, stop the bad behavior, enhance the debt collection ability of the bank and other ways to control risk, so VaR value will be reduced.

VaR model has the following advantages: it can be simple used to represent the size of the market risk with no technology, investors and managers with no professional background can judge financial risks by VaR value; it can be calculated in advance to reduce market risk, considering risk and return factors, and choosing a combination that can bring the greatest benefits with the same risk; and it also can determine the necessary regulatory capital and provide the supervision basis, and determine a scientific basis for the necessary capital to withstand market risks, financial institutions' capital can be established on the precise risk value, but also it can provide a scientific, consistent and fair standard for financial regulators to monitor the capital adequacy ratio of banks.

\section{System Functional Analysis}

The current credit risk is mainly divided into two kinds, namely credit risk and fraud risk. Credit risk is that the cardholder can not pay the number of paid bills to occur default; fraud risk includes credit card fraud, counterfeiting use, malicious overdraft and so on. For credit risk, the issuing bank can use rigorous credit check system to dynamically adjust the cardholder's credit limit for active risk management, so the loss of such risks can be avoided to the maximum extent. For fraud risk, the issuing bank can not take the initiative to prevent this risk, and the issuing bank will suffer huge losses if such risk occurs, which is the main control function of the system.

Working principle of credit card risk control system is that risk control model analyzes credit card transactions to find some suspicious transactions according to certain rules, and then subsequent manual analysis will be carried on to further confirm credit cards with risk transactions, the system will adopt appropriate measures, including get into contact with customer, reduce credit card limit, stop overdraft transaction temporarily, collect in advance and refuse to pay for 
sponsoring businesses and other measures, the activation of measures can reduce the credit card risk to achieve control and reduce losses.

The system need to understand three concepts, namely "customer", "account" and "user". "Customer" is credit card holder who applies information card from the financial institution; "account" has a certain format and structure to reflect the amount of credit card and their results. A credit card usually corresponds to an account, but when the main card and attached card are issued, the two share an account; "user" is the person using the system, including the cardholders, system administrators, business operators, data analysis members and management personnel of financial institutions.

In order to apply to the actual requirements of the credit card risk control system, the system should have the following features: in order to facilitate the maintenance of user information, users need to be assigned roles, and different roles correspond to different operating authorities; it is able to identify suspicious transactions rapidly based on pre-established risk control model, and track assign control tasks generated by certain rules; timely make the appropriate treatment to identified risks, including the cessation of trading, etc.; it can realize the query of related information in the process of risk control, such as credit card's cardholder information, sponsoring business information, issuer information and so on. Fig. 1 shows the system function.

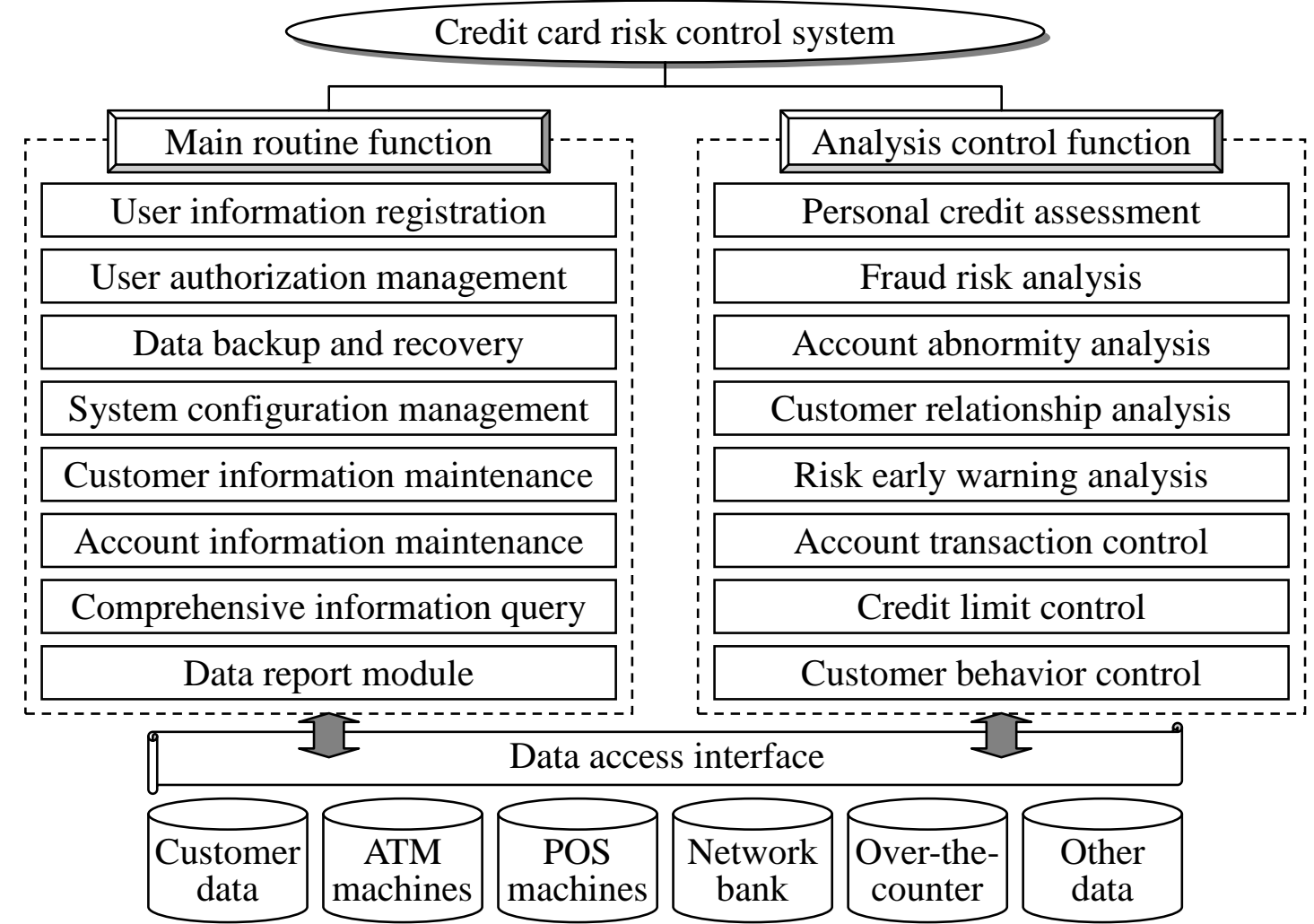

Fig. 1. Function on credit card risk control system

\section{Create Business Layer Components}

Layering is to achieve "high cohesion and low coupling." Use the idea of "divide and conquer", the problem can be divided into some small problems to be solved, it is easy to control, extend and allocate resources. Business layer has the core values of system architecture, playing a connecting role in data exchange between the data layer and the presentation layer, and it focuses on the development of business rules, realization of business process and system design related to business needs. The software components can be as a separate process, the purpose is to see components as "parts" to construct the software [4]. With the continuous development of software technology and the continuous improvement of software engineering, software components will be used as stand-alone software products on the market for developers to choose. There are many business 
layer components in the system, this article only creates business layer component of "credit card limit control".

(1) Data model: CreditLimitControlDataModel.

Data model is used to describe data manipulation includes what information, it is appropriate to package data information as attribute in theory, because the attribute not only can control read-write attribute, but also can be used for further processing. For rapid development, this article will define data members directly in Public form as follows:

\begin{tabular}{|l|l|}
\hline Public Class CreditLimitControlDataModel & Public c_bEffectSelection as Boolean \\
'Variable on data member of class & Public c_dEffectiveDate as Date \\
Public c_iId as Int64 & Public c_sOperatorName as String \\
Public c_sCustomerType as String & Public c_sOperatorNumber as String \\
Public c_sCustomerName as String & Public c_dOpeateDate as Date \\
Public c_sCardNumber as String & Public c_sRemarks as String \\
Public c_dCurrentLimit as Decimal & End Class \\
Public c_dCreditLimit as Decimal & \\
\hline
\end{tabular}

(2) Object model: CreditLimitControlObjectModel

In the .NET object model, all operations take the data model as unit, providing a range of operating data model methods, the object model more fully represents an object of the logic. "Credit card limit control" object model includes add, modify, delete, and query methods. The "add" is used to add a limit control record to the database; "modify" is used to modify a limit control record that already exists in the database; "delete" is used to delete a limit control record that already exists in the database; "query" is used to query limit control record that already exists in the database. Model code as follows: 


\begin{tabular}{|c|c|}
\hline & $\begin{array}{l}\text { // Update one record } \\
\text { Private Function UpdateOneLimit (ByRef rsData } \\
\text { As CreditLimitControlDataModel) As String } \\
\text { strSQL= "UPDATE CreditLimit Set } \\
\text { CustomerType = "' \& } \\
\text { rsData.c_sCustomerType \& "', } \\
\text { CustomerName = "' \& } \\
\text { rsData.c_sCustomerName \& "', CardNumber } \\
\text { = "' \& rsData.c_sCardNumber \& "', } \\
\text { CurrentLimit =" \& rsData.c_dCurrentLimit } \\
\text { \& ", CreditLimit = " \& } \\
\text { rsData.c_dCreditLimit \& ", EffectSelection = } \\
\text { " \& rsData.c_bEffectSelection \& ", } \\
\text { EffectiveDate = " \& rsData.c_dEffectiveDate } \\
\text { \& ", OperatorName = "' \& } \\
\text { rsData.c_sOperatorName \& "', } \\
\text { OperatorNumber = "' \& } \\
\text { rsData.c_sOperatorNumber \& "', OpeateDate } \\
\text { = " \& rsData.c_dOpeateDate \& ", Remarks = } \\
\text { "' \& rsData.c_sRemarks \& "' WHERE Id=" } \\
\text { \& rsData.c_iId } \\
\text { myCommand.Execute(strSQL) } \\
\text { If my.RecordCount=1 Then } \\
\text { Return "Update successfully" } \\
\text { Else } \\
\text { Reture "Update failed" } \\
\text { End If } \\
\text { End Function } \\
\text { // Delete one record } \\
\text { Private Function DeleteOneLimit (ByRef iId As } \\
\text { Int64) As String } \\
\text { Dim my as RecordSet } \\
\text { strSQL= "DELETE CreditLimit WHERE Id=" } \\
\text { \& iId } \\
\text { myCommand.Execute(strSQL) } \\
\text { If my.RecordCount=1 Then } \\
\text { Return " Delete successfully" } \\
\text { Else } \\
\text { Return " Delete failed" } \\
\text { End If } \\
\text { End Function } \\
\text { and }\end{array}$ \\
\hline
\end{tabular}

\section{Presentation Layer Implementation}

The presentation layer is a part of the business application program, which provides a communication mechanism between users and business services. Presentation layer design includes two aspects: the one is the presentation layer element. The presentation layer element is composed of the user component carried by GUI, it can be a Microsoft Windows form or Microsoft ASP.NET Web form, for more complex user interface, you can design user process component to coordinate UI elements and control user interactions; the other is the presentation layer design input. In the conception and planning stages, researched, analyzed and optimized information is the input of presentation layer, including the needs and constraints of solutions, usage scenarios, workflow model, user profiles and task descriptions, etc. [5]. 
The most important part in presentation layer is the user interface. For most users, the interface is the application. A well-designed user interface can help ensure the success of business applications and easily accepted by users. User interface components is responsible for managing the interactions with users, showing data to users, obtaining data from users, interpreting events triggered by a user operation, changing users interface state, and helping users to view the progress of the task. Specific functions of user interface components are: obtaining data from the user, and providing users with visual prompts, data efficacy and adequate control the mission to facilitate user input; capturing events from the user, and calling controller functions to notify the user interface components, starting a operation in current user process or changing the data of the current user to change the display data and method; limiting data type that user can input; performing data input validation, and limiting the scope of specific field values; mapping and transforming some values when information and infrastructure components work provided by user controls; formatting output values; localizing the presented data. The main controls and property settings of "Credit limit control" is shown in Table 1.

Table 1. Main controls and property settings

\begin{tabular}{c|c:c:c}
\hline Control name & Attribute & Attribute value & Explain \\
\hline frmCreditLimitControl(Form) & Caption & Credit limit control & Main interface form \\
\hline trvCustomerType (TreeView) & SelectedNode & Nothing & Display user type \\
\hline lstCustomers (ListBox) & Items & Qualified customers & Customer list \\
\hline lblCustomerName (Label) & Text & Customer Name & "Name" display \\
\hline txtCustomerName (TextBox) & ForeColor & VBRGB(255,0,0) & Cardholder name display \\
\hline txtCardNumber (TextBox) & Format & \#\#\#-\#\#\#\#-\#\#\#\#-\#\#\# & Credit card number \\
\hline txtCurrentLimit (TextBox) & Format & $\# \# \#$,\#\#\#,\#\#\#.\#\# & Current limit \\
\hline txtCreditLimit (TextBox) & Format & \#\#\#,\#\#\#,\#\#\#.\#\# & Credit limit \\
\hline rbnSelection1 (RadioButton) & Text & Effective immediately & Effective mode selection \\
\hline rbnSelection1 (RadioButton) & Checked & False & Specified date \\
\hline dtpEffectiveDate (DTPicker) & Format & dtpLongDate & Effective date \\
\hline btnControl (Button) & Text & OK & Quota control \\
\hline btnCancel (Button) & Text & Cancel & Cancel control \\
\hline
\end{tabular}

\section{Conclusion}

Credit card business is in a period of rapid development of the market, increasing the number of cards also bring risks. It is particular important to strengthen the credit card risk control [6]. The design and implementation of credit card risk control system in the paper can ensure sustained and healthy development of credit card business and maintain stability of financial sector.

\section{References}

[1] Emil J. Khatib, Raquel Barco, Ana Gómez-Andrades, et. al, "Data mining for fuzzy diagnosis systems in LTE networks," Expert Systems with Applications, vol. 42, no. 21, pp. 7549-7559, 2015.

[2] Chowdhury Farhan Ahmed, Nicolas Lachiche, Clément Charnay. et. al, "Flexible propositionalization of continuous attributes in relational data mining," Expert Systems with Application, vol. 42, no. 21, pp. 7698-7709, 2015.

[3] Véronique Van Vlasselaer, Cristián Bravo, Olivier Caelen, et. al, "APATE: A novel approach for automated credit card transaction fraud detection using network-based extensions," Decision Support Systems, vol. 75, no. 7, pp. 38-48, 2015. 
[4] Baidu Encyclopedia, "3-tier architecture," http://baike.baidu.com/link?url=9laQCwRDraXWRpaj3GbcI8z6gE4TegcmFlgPOQKEu_3p6 QDxOz5iS3cY8Ep3XIY8a1FytfKGOn6HH3410ivSq, 2016-2-5.

[5] Douding network, "Presentation layer design," http://www.docin.com/p-141357268.html, 2016-2-5.

[6] S. Gao, R. Chen, W. N. Gu, "Design and implementation of credit card risk precaution and watch system," Computer Engineering and Design, vol. 28, no. 18, pp. 4557-4560, 2007. 